(C) 1982. The Genetical Society of Great Britain

\title{
THE EFFECTS OF A CHROMOSOMAL INVERSION ON ADULT SIZE AND MALE MATING SUCCESS IN THE SEAWEED FLY, COELOPA FRIGIDA
}

\author{
R. K. BUTLIN, I. L. READ and T. H. DAY \\ Department of Genetics, University of Nottingham, University Park, \\ Nottingham, NG7 2RD
}

Received 4.iii.82

\section{SUMMARY}

\begin{abstract}
An association is reported between the $\alpha / \beta$ inversion polymorphism on chromosome I and adult size as assessed by the length of wings. $\alpha \alpha$ flies are larger than $\beta \beta$ flies, with heterokaryotypes intermediate, and the differences are more marked in males than in females. Laboratory mating experiments were performed in which a single female was given a choice of two males. By examining the genotypes of the progeny larvae, it is shown that the larger male is successful in a significantly greater proportion of trials than the smaller one. This mating success is dependent on the size difference between the males and on the female size. Together these observations suggest an indirect influence of the inversion on male mating success. The possible relevance of this effect to the maintenance of the inversion polymorphism in natural populations is discussed.
\end{abstract}

\section{INTRODUCTION}

MANY species of insects are polymorphic for chromosomal inversions. The most extensive studies of these polymorphisms have been in various species of Drosophila (da Cunha, 1955) especially $D$. pseudoobscura (Dobzhansky, 1971), D. persimilis (Spiess and Spiess, 1969; Yu and Spiess, 1978) and D. subobscura (Krimbas and Loukas, 1979).

It is clear from the Drosophila work that there exists a multitude of selection pressures that may influence the frequencies of inversions in natural populations. Bearing in mind that chromosomal inversions often involve tens or even hundreds of loci, it should be no surprise that inversions have multiple influences on the phenotype. The observed effects fall into two broad categories; effects on viability and survival, and effects on mating behaviour and fecundity.

In the seaweed fly, Coelopa frigida, approximately 25 per cent of the chromosomal bands are located within inversions (calculated from the data of Aziz, 1975). The existence of such a large proportion of the genome in the polymorphic state in natural populations (Philip, 1958) offers an ideal opportunity to study how genetic variability can be maintained on a wholesale scale.

The largest of these inversions, the $\alpha / \beta$ inversion on chromosome I (involving approximately 180 polytene chromosome bands), is polymorphic in all populations studied and the frequencies are remarkably constant both geographically and temporally (Butlin et al., 1982). This is strong indirect evidence that the polymorphism is selectively maintained.

Some of the selective forces acting on this inversion have been identified. Collins (1978) observed differences in egg to adult viability, with the $\alpha / \beta$ heterokaryotype having the highest survival. This heterosis increases greatly 
when the larvae are growing at high, though not unnatural, densities. Furthermore, there are differences in the egg to adult development times between genotypes that might also be expected to place the heterokaryotype at an advantage (Day et al., 1980).

Adult $C$. frigida are extremely variable in size (from about 3 to $9 \mathrm{~mm}$ in overall length), a fact that has caused taxonomic confusion in the past (Mayhew, 1939). In laboratory culture it was noticed that larger flies tended to eclose later than smaller ones, and with the known effect of the $\alpha / \beta$ inversion on development time, it seemed that adults of different karyotypes might differ in size. Since adult size is known to affect mating success in Drosophila (Ewing, 1961, 1964; Monclús and Prevosti, 1971) and other insects (Bryant, 1980), it seems possible that the inversion might have some influence on mating.

We report here correlations between karyotype, development time and size in animals from natural populations and show that under laboratory conditions, there is non-random mating with respect to the size of males. The possible relevance of this sexual selection to the maintenance of the $\alpha / \beta$ inversion polymorphism is discussed.

\section{MATERIALS AND METHODS}

Wild populations were sampled from the following three widely separated sites: St. Mary's Island on the north-east coast of England (Ordnance Survey map reference NZ 350753) in November 1979 and January 1980; Rustington on the south coast of England (TV 049014) in November 1979; and Morfa Nefyn on the north coast of Wales (SH 296408) in December 1980. Of the large St. Mary's Island sample collected in January 1980, only males were studied, as the females were required for another, unrelated, experiment.

The samples from Rustington and the first from St. Mary's Island were collected as larvae living in decomposing seaweed on the seashore (see Butlin et al., 1982). The larvae continued their development in the laboratory living on the seaweed in which they were collected. As the adults eclosed they were aspirated from the collecting cage and stored at $-20^{\circ} \mathrm{C}$ until required. The samples from Morfa Nefyn and the second from St. Mary's Island were collected as adults on the shore and were also stored at $-20^{\circ} \mathrm{C}$. The ecology and general biology of seaweed flies have been described by Egglishaw (1960) and Dobson (1974a, $b ; 1976)$.

\section{(i) Measurement of size}

Wing length was used as a measure of size because it is convenient, because it is strongly correlated with most other bodily dimensions, and because wing area is known to have a direct effect on courtship behaviour in Drosophila species (Ewing, 1964; Monclús and Prevosti, 1971). Each fly was measured using a binocular microscope at $\times 6$ magnification. The measurement used was the length from a prominent supra-alar bristle to the posterior margin of the longer wing in the folded position. Lengths are quoted in graticule divisions where 1 division $=1.45 \mathrm{~mm}$. After measurement the flies were examined for their chromosome I karyotype. 


\section{(ii) Karyotype analysis}

The karyotypes were inferred not from chromosomal squashes but by determination of their genotype at the alcohol dehydrogenase $(A d h)$ locus. The $A d h$ locus has been shown to be strongly associated with the $\alpha / \beta$ inversion system by Day et al. (1982). The $A d h-B$ allele is always associated with the $\alpha$ sequence and the $A d h-D$ allele with the $\beta$ sequence. In wild British populations a third allele, $A d h-C$, is present at a frequency of about $0 \cdot 05-0 \cdot 15$. This can be associated with either $\alpha$-or $\beta$-inversions. Since it is not possible to infer unambiguously the karyotype of animals carrying an $A d h-C$ allele, such animals have been omitted from this study.

The practical advantages of scoring animals by their Adh genotype, rather than directly from their karyotypes, far outweigh the errors introduced. Indeed, the method used may well be the more accurate one (see Day et al., 1982). Homogenisation of animals, starch gel electrophoresis and staining of gels for ADH activity have been described by Butlin et al. (1982).

\section{(iii) Mate-choice experiments}

Animals used were from stock lines maintained in the laboratory for several years. One was fixed for the $\alpha$ sequence and carried the $A d h-B$ allele, and the other was fixed for the $\beta$ sequence with the $A d h-D$ allele. Day and Buckley (1980) have described the techniques of laboratory culture of $C$. frigida but in the experiments described here the food was slightly modified by omission of powdered milk and of the strips of Laminaria on the surface of the medium.

Virgin males and females were obtained by removing all flies twice daily from stock cages and separating the sexes immediately. At the normal culture temperature of $26^{\circ} \mathrm{C}$, males do not mate until approximately 18 hours, and females 20 hours, after eclosion (Thompson, 1951). Flies were then stored until required at $4^{\circ} \mathrm{C}$ in bottles with cotton wool wetted with 0.5 per cent mannitol solution.

In outline the experiment consisted of offering one female a choice of two genetically different males, and then analysing the progeny to determine which of the males was the father. In each trial a single virgin female and two virgin males that had eclosed on the same day were placed in a glass vial $(8 \times 2.5 \mathrm{~cm})$ containing about $1 \mathrm{~cm}$ depth of culture medium. The males, one from the $\alpha \alpha$ stock and the other from the $\beta \beta$ stock, were chosen so that one was distinctly larger than the other. The vials were kept in constant darkness at $26^{\circ} \mathrm{C}$ and observed daily. When the first egg batch appeared, the three adults were removed and their wing lengths recorded. Any vial in which one of the adults had died was discarded. Larvae hatching in the vials were transferred to larger canisters $(7 \times 7.5 \mathrm{~cm}$ diam.) containing culture food, and after about six days third instar larvae were collected. The Adh genotypes of four larvae, or in some cases only two, were determined by gel electrophoresis.

\section{Results}

(i) Sizes and genotypes of wild flies

The sizes and genotypes of animals collected either as larvae or as adults from three widely separated populations are given in figs. 1a and 
MALES

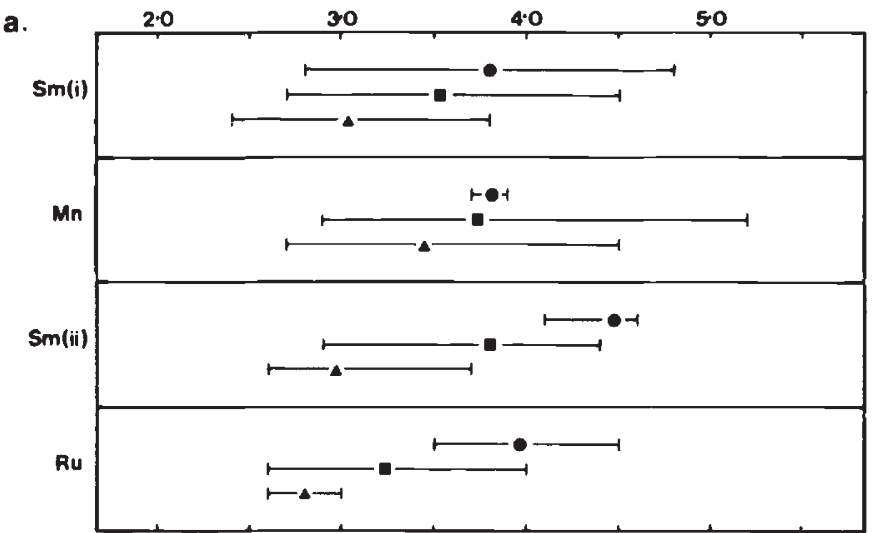

SAMPLE VARIANCE

SIZE RATIO

a.

b.

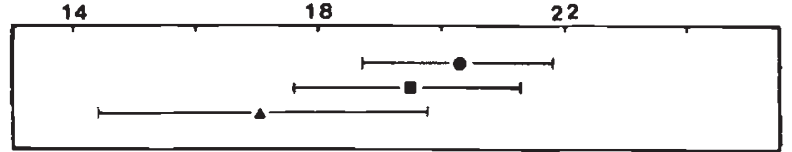

18

22

\section{FEMALES}

c.

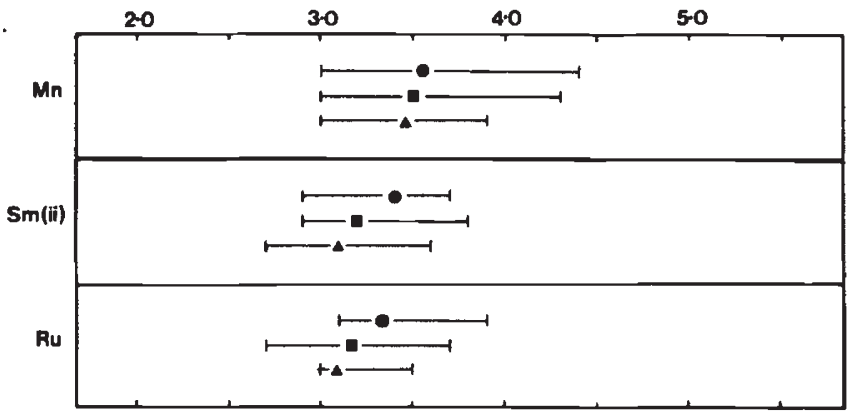

d.

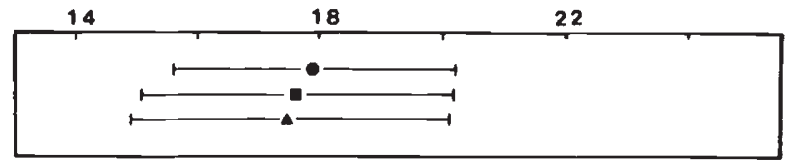

FIG. 1.-Wing lengths and development times of Coelopa frigida. The mean wing lengths, with observed ranges, for each genotype are shown in (a) males and (c) females. The units are graticule divisions - see the Materials and Methods s..tion. The mean development times of males (b) and females (d) are taken from Day et al. (1980). The times are expressed in days and the bars represent one standard deviation.

Sample collection sites:

Sm(i) St. Mary's Island, January 1980

Mn Morfa Nefyn

Sm(ii) St. Mary's Island, November 1979

$\mathrm{Ru} \quad$ Rustington

Chromosome I genotypes:,$\alpha \alpha ; \square, \alpha / \beta ; \Delta, \beta \beta$. 
1c. Several obvious points emerge. First, in every case the $\alpha \alpha$ homozygotes are the largest, $\beta \beta$ homozygotes the smallest and the $\alpha / \beta$ heterozygotes intermediate. In most populations if the sexes are analysed separately these genotypic differences are significant. Secondly the females are far more homogeneous in size, and are slightly smaller than the males. Thirdly the mean size varies between populations. In laboratory culture, Collins (1978) has shown that mean adult size is very dependent on larval density, animals growing at high density eclosing into much smaller adults. Although no estimate of larval density was made in the populations studied here, it seems very likely that this factor is the important one. Lastly, during this study some animals carrying the $A d h-C$ allele were identified and measured (data not shown). They were extremely variable both within and between populations. This was expected since all three genotypes $(A d h-B / C, A d h$ $C / D$ and $A d h-C / C)$ are heterogeneous with respect to the $\alpha / \beta$ inversion (Day et al., 1982) and there may even be more than one $A d h-C$ allele (see Day and Buckley, 1980). The only consistency is that $A d h-B / C$ heterozygotes are larger than $A d h-C / D$ heterozygotes.

The most striking feature of these data is that in spite of the populations coming from different parts of the country (each is separated by at least $850 \mathrm{~km}$ of coastline), and of the mean sizes varying between populations, there are clear and consistent differences between genotypes, particularly in the males.

\section{(ii) Sizes and development times of wild flies}

Day et al (1980) showed that there are genotypic differences in development rates and it can be seen from figs. $1 \mathrm{~b}$ and $1 \mathrm{~d}$ that there is a strong correlation between development rate and adult size. The order of genotypes is the same in both sexes and females are more homogeneous in their development rate as well as their size.

It may be that size differences between genotypes are a direct result of differences in rates of development. This seems likely as it is a common observation in holometabolous insects that animals spending longer in the major eating phase of the life cycle-in this case the second and third larval instars-end up as larger adults. However, it is also possible that the $\alpha / \beta$ inversion has a direct effect on adult size, independently of its effect on development rate. Furthermore there are certainly environmental influences on both development rate and adult size. For example Collins (1978) found that increasing larval density results in slower development and smaller adults.

We suggest then that the $\alpha / \beta$ inversion, by a combination of direct and indirect effects (e.g., by influencing egg to adult development), is a major determinant of the size of adults.

\section{(iii) Effect of adult size on mate choice}

Differences in adult size must surely have many consequences on the chances of survival of animals (feeding requirements, longevity, etc.). However in many insects the adult stage of the life cycle is largely concerned with reproduction. 
In our search for selective pressures acting on the $\alpha / \beta$ inversion polymorphism we have (so far) concentrated on one aspect of reproductionmale mating success. Our initial approach has been to offer a female a choice of two males and determine which is successful.

In a series of trials of this type we have measured several variables: size and genotype of the female, size and genotype of each male, which male actually fathered the progeny and how many times both males contributed to the progeny. The most informative method of analysis would be an analysis of $\chi^{2}$ which would reveal not only first order effects but more complex interactions between the variables. With so many variables the scale of the experiment would need to be impracticably large-far more than the 350 trials that were actually carried out. We will therefore analyse the variables, two or three at a time, in order to highlight the principal effects without attempting to identify more complex interactions.

It should also be noted that males were selected to be large or small, or in some cases as nearly the same as possible. The size distributions do not therefore represent the distributions normally seen for each genotype, but the range of sizes does fall within the range observed in natural populations.

(a) Is the larger male more successful than the smaller one?

Out of 257 trials in which only one male fathered the progeny, 200 were fathered by the large male. This is a highly significant departure from randomness $\left(\chi^{2}=79 \cdot 6, p<0 \cdot 001\right)$.

(b) How similar do the males need to be before the mating is random?

When the data are grouped in terms of size differences between the two competing males (table 1), mating is random when the males are within

TABLE 1

Relationship between the success of the larger male and the difference in size between the two males

$\begin{array}{lccccc}\begin{array}{l}\text { Difference in size between } \\ \text { males }\end{array} & <0.2 & 0.3-0.5 & 0.6-0.8 & 0.9-1.1 & >1.2 \\ \begin{array}{l}\text { No. of trials } \\ \text { \% egg batches fathered by }\end{array} & 25 & 62 & 79 & 66 & 25 \\ \text { larger male } & 52 & 71 & 86 & 82 & 84\end{array}$

Sizes are expressed in graticule units (see Materials and Methods). The mating in the smallest size difference class is random but in all other classes it departs significantly from randomness (in every case $\chi_{1}^{2}>10.9, p<0.001$ ).

0.2 units of each other. This corresponds to a difference between the males of 5-10 per cent in their wing size. When the difference between them exceeds approximately 10 per cent the mating becomes non-random. The advantage to the larger males increases until a plateau is reached in the $0 \cdot 6-0 \cdot 8$ class (corresponding approximately to a 25 per cent difference in size) when over 80 per cent of egg batches are fathered by the larger male. 
(c) What is the effect of male size on mating success?

If we consider only those trials where the difference between males is greater than 0.6 units (i.e., on the plateau of size differences-see table 1) then we can enquire whether the larger male enjoys greater success when he himself is large than when he is small. There is no clear evidence that this is so (table 2). Neither is there any evidence that males can be so large that their size has become a disadvantage.

\section{TABLE 2}

Relationship between the success of the larger male and his size for trials where difference in size between males is $\geqslant 0.6$

$\begin{array}{lcccc}\text { Size of the larger male } & <3 \cdot 3 & 3 \cdot 4-3 \cdot 5 & 3 \cdot 6-3 \cdot 7 & >3 \cdot 8 \\ \text { No. of trials } & 39 & 46 & 51 & 34 \\ \% \text { egg batches fathered by } & 82 & 91 & 78 & 88\end{array}$

larger male

Sizes are expressed in graticule units. There is no significant correlation between male success and the size of the larger male $(b=0 \cdot 02, p=0 \cdot 8)$.

(d) What is the effect of female size on male mating success?

From table 3 it can be seen that the advantage to the larger male increases as the size of the female increases. This effect is very much more pronounced in females with the $\beta \beta$ genotype. If the female plays a relatively passive role in courtship then it is not at all clear why large males should have greater mating success with large rather than small females. Alternatively if the female is playing a more active role then perhaps large females show a preference for large males (see (e) below). This of course would be assortative mating with respect to size, a phenomenon that is familiar in many species including man.

TABLE 3

Relationship between the success of the larger male and female size $\%$ of trials in which the larger male was successful

$\begin{array}{cccc}\text { Female size } & \alpha \alpha \text { females } & \beta \beta \text { females } & \begin{array}{c}\text { (regardless of } \\ \text { female genotype) }\end{array} \\ <2 \cdot 7 & 70(46) & 71(17) & 70(63) \\ 2 \cdot 8-2 \cdot 9 & 67(27) & 81(21) & 73(48) \\ 3 \cdot 0-3 \cdot 1 & 77(35) & 80(20) & 78(55) \\ 3 \cdot 2-3 \cdot 3 & 85(34) & 83(23) & 84(57) \\ >3 \cdot 4 & 71(7) & 96(27) & 91(34)\end{array}$

The number of trials in each size class is given in brackets. Sizes are expressed in graticule units.

There is no significant correlation between success of the larger male and female size with $\alpha \alpha$ females $(b=0.05, p=0.6)$; but there is with $\beta \beta$ females $(b=0 \cdot 13, p=0 \cdot 02)$, and when the genotype of the female is disregar$\operatorname{ded}(b=0.13, p=0.003)$.

(e) Is it the male nearest to the female's size who is successful?

Out of 237 trials where only one male was successful, and where the female was not exactly intermediate in size between the two males, the 
male nearer in size was successful 119 times and the one more distant in size 118 times. No statistical test is needed to conclude that there is no assortative mating such that matings tend to be between animals of similar size.

\section{(f) How commonly are both males successful?}

Out of 302 trials there were 45 in which both males contributed to the offspring. It is possible that two egg batches had been laid before the adults were removed and that they had been fathered by different males. This is unlikely since females very rarely lay two batches within 24 hours. Usually the interval between egg laying is 48 hours. We think it is more likely that, contrary to the observations of Thompson (1951) and Burnet (1961) who presented the males one after the other, batches can be of mixed fatherhood. If this is so then the small number of offspring used to determine fatherhood will have introduced an error into the observations. Some progeny of mixed fatherhood will have been attributed to only one of the males. However, only if a particular class of males consistently fathered more than half of the offspring would the results be biased. In this case it is possible that some aspects of male fecundity (such as sperm number or viability) have become confounded with our measure of male mating success.

84 per cent of mixed batches were laid by females with the $\alpha \alpha$ genotype and only 16 per cent by $\beta \beta$ females. This genotypic difference between females is statistically significant $\left(\chi^{2}=11 \cdot 1, p<0 \cdot 01\right)$ and may result from some genetic difference in the mating behaviour of females, such as a shorter time to remating in $\alpha \alpha$ females. Pyle and Gromko (1981) have shown that the interval between matings is under genetic control in Drosophila melanogaster.

\section{(g) Is there any effect of inversion karyotype on mating success?}

Table 4 shows the data from all combinations of genotypes. When setting up these trials it proved difficult to select $\beta \beta$ males as large as the large $\alpha \alpha$ males. Consequently the $\alpha \alpha$ males tended to be larger than $\beta \beta$

TABLE 4

Interaction between the success of the larger male and genotypes of the males and female

$\begin{array}{lc}\begin{array}{l}\text { Genotype of } \\ \text { larger male }\end{array} & \begin{array}{c}\% \text { of trials in } \\ \text { which the larger } \\ \text { and female }\end{array} \\ \text { male was successful }\end{array}$

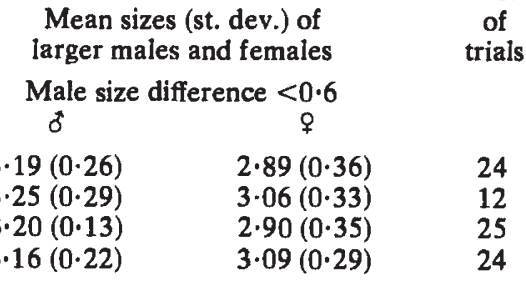

$\begin{array}{ccc}\delta & q & \\ \alpha \alpha & \alpha \alpha & 74 \\ \alpha \alpha & \beta \beta & 92 \\ \beta \beta & \alpha \alpha & 54 \\ \beta \beta & \beta \beta & 56\end{array}$

$\begin{array}{lll}\alpha \alpha & \alpha \alpha & 81 \\ \alpha \alpha & \beta \beta & 97 \\ \beta \beta & \alpha \alpha & 76 \\ \beta \beta & \beta \beta & 84\end{array}$

Male size difference $>0.6$ 
males. In addition $\alpha \alpha$ females tended to be smaller than $\beta \beta$ females. These genotypic differences in size make it more difficult to separate the effects of genotype and size.

The combination of large $\alpha \alpha$ male and $\beta \beta$ female stands out from other genotypic combinations. If we suppose firstly that large females show a greater tendency to be fertilized by the larger male than do small females (for which there is some support-see (d) above), and secondly that large males tend to be more successful than small males (which may be reasonable, but for which there is no support-see (c) above), then these two effects might combine to give the exceptionally high mating preference seen with large $\alpha \alpha$ males and $\beta \beta$ females. In other words this exceptional mating performance seen with this combination of genotypes might very largely be a result of size rather than genotype. Nevertheless we still think there is some evidence that $\beta \beta$ females exhibit a particularly strong mating preference for $\alpha \alpha$ males. There are two lines of evidence that support this. When $\beta \beta$ females are compared with $\alpha \alpha$ females of similar size, the former show a stronger preference for the large male (see table 3 ). Secondly, when the males offered are similar in size (i.e., the difference in size between males is less than 0.6 -table 4 ) the $\beta \beta$ females still exhibit a near perfect preference ( 92 per cent) for the large male if he carries the $\alpha \alpha$ genotype, but negligible preference ( 56 per cent) if he carries the $\beta \beta$ genotype.

If genotypic mating preferences exist independently of size effects, then they are clearly not very obvious. Further experiments specifically designed to reveal such effects are necessary before we can be sure that the $\alpha / \beta$ genotype directly affects mating preference. There can be little doubt, however, that the outcome of competition between males for a mate is largely determined by the relative sizes of the males. Since the $\alpha / \beta$ inversion is a major determinant of male size (fig. 1) we suggest that there may well be an indirect effect of this inversion on mating success.

\section{Discussion}

We have presented evidence that the $\alpha / \beta$ inversion on chromosome I may have an influence on mating and we propose that the relationship is indirect. Day et al. (1980) identified a karyotypic effect on the egg to adult development time. We have demonstrated a strong correlation between development time and adult size, which is likely to be one of the reasons for the genotypic differences in size observed in wild flies. In laboratory trials in which two males are competing for a female, there is a strong advantage to the larger male. We have yet to show that this chain of relationships can give rise to non-random mating in natural populations.

Two general points are raised by these results. The first concerns the behavioural mechanism by which large males gain an advantage in mating, and the second to what extent non-random mating is likely to occur in natural populations.

The interpretation of non-random mating in Coelopa depends very much on the relative importance of male competition and of female discrimination. At one extreme we could imagine the female being passive and being mated by the victor of some sort of male fight, as seems to happen in dung flies (Parker, 1970). At the other extreme perhaps the males are more or less passive and are chosen on the basis of size, or 
perhaps noise, by the female. In many insects there is an elaborate interaction between males and females during courtship (see Matthews and Matthews, 1978). In D. melanogaster larger males tend to be more successful in courtship, apparently because they sing more loudly, and as a result are more stimulating to the female (Bastock and Manning, 1955; Ewing, 1961, 1964).

In Coelopa preliminary observations suggest that courtship is far less elaborate. Our initial impression is that in our trials, movement of the three animals is random, and when a male comes close to a female he almost immediately mounts her. If the two males meet then often one attempts to mount the other. The outcome of either of these encounters is that the mounting male may be kicked off (particularly if he has mounted the other male), or he may proceed to tap the partner on the head with his fore-legs. An advantage may be gained by the larger male either if he is more active, and therefore takes part in more encounters, or is less readily kicked off by the female. We consider the latter alternative the likely one, though clearly more detailed observation is required to confirm this.

There are several reasons why it is premature to extrapolate the laboratory results to flies living in the wild. The mating trials were performed with inbred laboratory stocks in a confined space in constant darkness. This must be both unnatural and an oversimplification. For example, we do not know how often male-female encounters are between two, three, or more animals, and it will prove exceedingly difficult to find out on the seashore. Furthermore the genotypes of animals available to mate may well vary from day to day. The fact that genotypes develop at different rates means that the first females to eclose are more likely to be mated by the fast developing $\beta \beta$ males (Day et al., 1980). Later in the sequence of eclosion, the genotypes of males available to mate will change in favour of the more slowly developing $\alpha \alpha$ homozygotes. How this changing availability of genotypes will interact with differences in mating success due to size is difficult to predict. A further complication is that wrack beds may be swept out to sea and replaced by a fresh batch of seaweed at varying times in the eclosion sequence. If the wrack bed is replaced early then the $\beta \beta$ homozygotes might be at a reproductive advantage because they develop quickly, even though they may be very small animals. If the wrack bed is longer lived then perhaps the $\alpha \alpha$ males will have more mating success because they are large. We are currently attempting to measure mating success in wild flies under conditions of changing genotype frequencies.

Multiple insemination with displacement of a proportion of the earlier male's sperm by that of the subsequent male is yet another complication. Its effects in other species of insects have been reviewed by Boorman and Parker (1976) and Prout and Bundgaard (1977). In Coelopa, multiple insemination is not uncommon, and there is the suggestion that females of different chromosome I karyotype, differ in their propensity to remate. Bearing in mind that females seem to require little stimulation from the males, multiple insemination may be yet another factor influencing the $\alpha / \beta$ polymorphism.

The observation that inversions affect various aspects of mating is not new. Duration of copulation in D. persimilis (Kaul and Parsons, 1965), female receptivity in $D$. pavani (Yu and Spiess, 1978) and mating speed 
in D. subobscura (Monclús and Prevosti, 1971), D. persimilis (Spiess and Spiess, 1967, 1969), D. pseudoobscura (Spiess and Langar, 1964) and D. pavani (Brncic and Koref-Santibanez, 1964), have all been shown to be influenced by chromosomal inversions.

The effect of the $\alpha / \beta$ inversion on multiple insemination and mating success of large males in Coelopa frigida, provides another example of inversions influencing reproductive performance. The job of disentangling and understanding the selective forces acting on an inversion that includes approximately 8 per cent of the genome will surely be a difficult one.

Acknowledgments. - This work was supported by a studentship (to R.K.B.) from the Science Research Council, whose help is gratefully acknowledged.

\section{REFERENCES}

AzIZ, J. B. 1975. Investigations into Chromosomes 1, 2 and 3 of Coelopa frigida. Ph.D. Thesis. University of Newcastle upon Tyne.

BASTOCK, M., AND MANNING, A. 1955. The courtship of Drosophila melanogaster. Behaviour, 8, 85-111.

BOORMAN, E., AND PARKER, G. A. 1976. Sperm (ejaculate) competition in Drosophila melanogaster, and the reproductive value of females and males in relation to female age and mating status. Ecol. Entomol, 1, 145-155.

BRNCIC, D., AND KOREF-SANTIBANEZ, s. 1964. Mating activity in homo- and heterokaryotypes in Drosophila pavani. Genetics, 49, 585-591.

BRYANT, E. H. 1980. Geographic variation in components of mating success of the house fly, Musca domestica L., in the United States. Am. Naturalist, 116, 655-669.

BURNET, B. 1961. On the distribution of recessive embryonic lethals in a natural population of Coelopa frigida (Fab). Genet. Res. Camb., 2, 249-271.

BUTLIN, R. K., COLLINS, P. M., SKEVINGTON, S. J., AND DAY, T. H. 1982. Genetic variation at the alcohol dehydrogenase locus in natural populations of Coelopa frigida. Heredity, 48, 45-55.

COLlins, P. M. 1978. Studies on genetic polymorphism in Coelopa frigida. Ph.D. Thesis. University of Nottingham.

DA CUNHA. A. B. 1955. Chromosomal polymorphism in the Diptera. Advances in Genetics, 7, 93-138.

DAY, T. H., AND BUCKLEY, P. A. 1980. Alcohol dehydrogenase polymorphism in the seaweed fly, Coelopa frigida. Biochem. Genet., 18, 727-742.

DAY, T. H., DOBSON, T., HILlIER, P. C., PARKIN, D. T., AND CLARKE, B. 1980. Different rates of development associated with the alcohol dehydrogenase locus in the seaweed fly, Coelopa frigida. Heredity, 44, 321-326.

DAY, T. H., DOBSON, T., HILlIER, P. C., PARKIN, D. T., AND CLARKE, B. 1982. Non-random association of enzyme and chromosome polymorphisms in the seaweed fly, Coelopa frigida. Heredity, 48, 35-44.

DOBSON, T. 1974a. Studies on the biology of the kelp fly Coelopa in Great Britain. J. Nat. Hist., 8, 155-177.

DOBSON. T. 1974b. Mechanisms controlling species composition in natural populations of the seaweed fly Coelopa. J. Nat. Hist., 8, 653-673.

DOBSON, T. 1976. Seaweed flies (Diptera: Coelopidae, etc.). In Cheng. L. (ed.) Marine Insects, North-Holland Publishing Company.

DOBZHANSKY, T. 1971. Evolutionary oscillations in Drosophila pseudoobscura. In R. Creed, (ed.) Ecological Genetics and Evolution, Blackwell, Oxford.

EGglishaw, H. J. 1960. Studies on the family Coelopidae (Diptera). Trans. R. ent. Soc. Lond., 112, 109-140.

EWING, A. 1961. Body size and courtship behaviour in Drosophila melanogaster. Anim. Behav., 9, 93-99.

EWING, A, 1964. The influence of wing area on the courtship behaviour of Drosophila melanogaster. Anim. Behav., 12, 316-320. 
KAUL, D., AND PARSONS, P. A. 1965. The genotypic control of mating speed and duration of copulation in Drosophila pseudoobscura. Heredity, 20, 381-392.

KRIMBAS, C. B., AND LOUKAS, M. 1979. The inversion polymorphism of Drosophila subobscura. Evol. Biol., 12, 163-234.

MATTHEWS, R. W., AND MATTHEWS, J. R. 1978. Insect. Behavior. John Wiley and Sons, New York.

MAYHEW, A. W. 1939. Studies on the structure and life history of shore flies of the genus Coelopa. M.Sc. Thesis. University of Durham.

MONCLÚS, M., AND PREVOSTI, A. 1971. The relationship between mating speed and wing length in Drosophila subobscura. Evolution, 25, 214-217.

PARKER, G. A. 1970. Sperm competition and its evolutionary consequences in the insects. Biol. Rev., 45, 525-568.

PHILIP. U. 1958. Genetics and cytology of the seaweed fly Coelopa frigida (Fab). Proc. Xth. Congr. Genet., Montreal, p. 217.

PROUT, T., AND BUNDGAARD, J. 1977. The population genetics of sperm displacement. Genetics, 85, 95-124.

PYLE, D. W., AND GROMKO, M. H. 1981. Genetic basis for repeated mating in Drosophila melanogaster. Am. Naturalist, 117, 133-146.

SPIESS, E. B., AND LANGAR, B. 1964. Mating speed control by gene arrangements in Drosophila pseudoobscura homokaryotypes. Proc. Natl. Acad. Sci. U.S., 51, 1015-1019.

SPIESS, E. B., AND SPIESS, L. D. 1967. Mating propensity, chromosomal polymorphism and dependent conditions in Drosophila persimilis. Evolution, 21, 672-678.

SPIESS, E. B., AND SPIESS, L. D. 1969. Mating propensity, chromosomal polymorphism and dependent conditions in Drosophila persimilis II. Factors between larvae and between adults. Evolution, 23, 225-236.

THOMPSON, U. 1951. Studies on the genetics and ecology of Coelopa frigida (Fab). Ph.D. Thesis. University of Durham.

YU, H. F., AND SPIESS, E. B. 1978. Chromosomal polymorphism and female receptivity in a natural population of Drosophila persimilis. Genetics, 90, 783-800. 